\title{
An Inverse Problem Solution for Thermal Conductivity Reconstruction
}

\author{
TCHAVDAR T. MARINOV, ROSSITZA S. MARINOVA \\ Department of Natural Sciences \\ Southern University at New Orleans \\ 6400 Press Drive, New Orleans, LA 70126 \\ USA \\ Department of Mathematical and Physical Sciences \\ Concordia University of Edmonton \\ 7128 Ada Blvd, Edmonton, AB, T5B 4E4

\section{CANADA}

\begin{abstract}
This work deals with the inverse problem of reconstructing the thermal conductivity coefficient of the $(2+1) \mathrm{D}$ heat equation from over-posed data at the boundaries. The proposed solution uses a variational approach for identifying the coefficient. The inverse problem is reformulated as a higher-order elliptic boundary-value problem for minimization of a quadratic functional of the original equation. The resulting system consists of a well-posed fourth-order boundary-value problem for the temperature and an explicit equation for the unknown thermal conductivity coefficient. The existence and uniqueness of the resulting higher-order boundary-value problem are investigated. The unique solvability of the inverse coefficient problem is proven. The numerical algorithm is validated and applied to problems of reconstructing continuous nonlinear coefficient and discontinuous coefficients. Accurate and stable numerical solutions are obtained.
\end{abstract}

Key-Words: Thermal conductivity; Inverse problem; Variational method

Received: January 12, 2021. Revised: July 5, 2021. Accepted: July 19, 2021. Published: July 23, 2021.

\section{Introduction}

There are many practically important problems involving parabolic partial differential equations, which are classified as inverse in the sense that the way they are posed is not the standard initial, boundary, or initial-boundary value for which the correctness and well-posedness of the problem has been proved. Clearly, the incorrect problems are inverse in this sense. The first example for an incorrect problem was shown by [6] for the initial value problem for Laplace equation (the so-called "analytical continuation" problem).

The class of inverse problems is wider than the class of incorrect problems since the former contains also the problems involving an unknown coefficient in the governing equation to be estimated from additional (overposed) data at the boundary or at some internal points (lines, surfaces) in the domain. In this case, the problem is inverse in the sense that no explicit equation is available to evaluate the unknown coefficient. Intuitively speaking, one should adjust the coefficient in order to make solution meet the overposed data at the boundaries.

The problem for thermal conductivity coefficient has attracted attention recently, see [1, 2, 5, 7, 8, 10, 11, 12, 14, 18, 21].

The coefficient identification may or may not be incorrect depending on existence and uniqueness of the solution. In most of the situations these questions are hard to answer and any kind of approach capable to shed some light on the problem is welcome. Even if an abstract theorem of uniqueness is available, the actual computation of the unknown coefficient still remains a formidable difficulty and the quest for effective algorithms is still on. At the same time, the estimation of an unknown coefficient from overposed boundary data is of significant practical importance when creating non-invasive methods to identify the material properties of a continuum.

Common approach to the solution of inverse problems is based on regularization methods (see, e.g., [4, 13, 20]), which requires finding the minimum with respect to a parameter (called a regularization parameter) of a properly constructed functional. In general, the numerical solution of the regularization algorithm does not always approach the exact solution when the regularization parameter becomes very small.

In this work we apply the so-called Method of Variational Imbedding (MVI) to solve the inverse problem for thermal conductivity reconstruction. A comparison between the regularization with respect to a small parameter and MVI, applied to the same problem, shows the advantages of the latter. Both methods come up with a numerical procedure for solving higher order equations. The MVI gives a solution of the original problem that coincides with the solution of the variational problem. 


\section{The Direct Problem}

This work deals with thermal conductivity identification from the heat conduction equation satisfying specific boundary and initial conditions. The general differential equation of heat conduction [9] within a solid is:

$$
\frac{\partial T(\vec{r}, t)}{\partial t}=\nabla[\lambda \nabla T(\vec{r}, t)]+q(\vec{r}, t),
$$

where the variables are: $\vec{r}-$ the position vector within the body; $t$ - the time; $T$ - the temperature at any point $\vec{r}$ and any time $t ; \lambda$-the thermal conductivity; $q-$ the heat generation in the medium.

We consider the initial-boundary value problem for the heat conduction in a bounded domain. The two-dimensional heat conduction equation is

$$
\frac{\partial T}{\partial t}=\frac{\partial}{\partial x}\left(\lambda \frac{\partial T}{\partial x}\right)+\frac{\partial}{\partial y}\left(\lambda \frac{\partial T}{\partial y}\right),
$$

where $\Omega=\{(x, y): 0<x<1 ; 0<y<1\} \subset R^{2}$ is the domain, and $T=T(x, y, t)$. There are no internal heat sources, i.e. $q(x, y, t)=0$.

In this work, the thermal conductivity is considered to be a function of time $t$, i.e., $\lambda=\lambda(t)$. Under this assumption, equation (2) reduces to

$$
\begin{aligned}
& \mathcal{R}(x, y, t):=-\frac{\partial T(x, y, t)}{\partial t} \\
& +\lambda(t) \frac{\partial^{2} T(x, y, t)}{\partial x^{2}}+\lambda(t) \frac{\partial^{2} T(x, y, t)}{\partial y^{2}}=0 .
\end{aligned}
$$

If the coefficient $\lambda=\lambda(t)$ is known, the problem can be solved provided that an initial condition and a boundary condition at each boundary point $(x, y) \in$ $\partial \Omega$ of the body are available, namely

$$
\begin{aligned}
T(x, y, 0) & =\phi(x, y), \\
\left.T(x, y, t)\right|_{(x, y) \in \partial \Omega} & =\psi(x, y, t) .
\end{aligned}
$$

The system of equations (3)-(5) comprises the socalled direct problem.

\section{The Inverse Problem}

We now introduce the inverse problem considered in this paper. Suppose that the thermal conductivity coefficient $\lambda(t)$ is not known. In order to identify it, we need more information for the temperature $T(x, y, t)$. We assume that the temperature field is not only known in the initial time moment $t=0$, but also in a specified final time moment, $t=t_{F}$,

$$
T\left(x, y, t_{F}\right)=\Phi(x, y) .
$$

We also assume that the heat flux associated with a temperature profile $T$ is given for all $t \in\left[0, t_{F}\right]$ and $(x, y) \in \partial \Omega$, i.e.,

$$
-\left.\lambda \frac{\partial T}{\partial \nu}\right|_{(x, y) \in \partial \Omega}=\Psi(x, y, t),
$$

where $\frac{\partial T}{\partial \nu}$ is the normal derivative of the temperature function $T$ at the boundary point $(x, y) \in \partial \Omega$. If the thermal conductivity $\lambda(t)$ is known, the problem (3)-(7) is over-determined. If the function $\lambda(t)$ is not known, it is possible to find the temperature $T$ and the thermal conductivity simultaneously. The pair of functions $(T, \lambda)$ is a solution of the problem (3)-(7). These type of problems are inverse problems.

\subsection{Variational Approach to Solving the Inverse Problem}

Following the idea of MVI, [3, 15, 16, 17], we replace the problem (3)-(7) with a minimization problem for the quadratic functional of the original equation

$$
\mathcal{I}(T, \lambda)=\int_{0}^{t_{F}} \iint_{\Omega} \mathcal{R}^{2} d x d y d t \longrightarrow \min ,
$$

under the conditions (4)-(7).

The functional $\mathcal{I}(\bar{T}, \lambda)$ is a quadratic and homogeneous function of its argument $\mathcal{R}(x, y, t)$. Hence, it attains its minimum if and only if the argument is zero, i.e. $\mathcal{R}(x, y, t)=0$. It follows that there is an one-to-one correspondence between the solution of the original problem (3), (4)-(7) and the minimization problem (8), (4)-(7).

\subsection{The "Imbedded" Boundary-Value Problem}

Necessary conditions for minimization of the functional $\mathcal{I}(T, \lambda)$ are derived from the Euler-Lagrange equations for the functions $T(x, y, t)$ and $\lambda(t)$. The equation for the function $T(x, y, t)$ is

$$
\begin{aligned}
\left(\frac{\partial}{\partial t}+\lambda \frac{\partial^{2}}{\partial x^{2}}+\lambda \frac{\partial^{2}}{\partial y^{2}}\right) & \\
\left(-\frac{\partial T}{\partial t}+\lambda \frac{\partial^{2} T}{\partial x^{2}}+\lambda \frac{\partial^{2} T}{\partial y^{2}}\right) & =0
\end{aligned}
$$

If the functions $T$ and $\lambda$ are as many time differentiable as needed, the equation (9) becomes

$$
\begin{array}{r}
-\frac{\partial^{2} T}{\partial t^{2}}+\lambda^{2}\left(\frac{\partial^{4} T}{\partial x^{4}}+\frac{\partial^{4} T}{\partial y^{4}}+2 \frac{\partial^{4} T}{\partial y^{2} \partial x^{2}}\right) \\
+\frac{d \lambda}{d t}\left(\frac{\partial^{2} T}{\partial x^{2}}+\frac{\partial^{2} T}{\partial y^{2}}\right)=0 .
\end{array}
$$


Equation (10) is of the second order with respect to the time $t$ and of the fourth order with respect to the spatial variables $x$ and $y$. There exist sufficient number of initial and boundary conditions (4) $-(7)$ for solving (10). The problem is well-posed if the function $\lambda(t)$ is known. Hence, using the MVI, the inverse problem is embedded into a higher-order but well-posed elliptic boundary-value problem. Note that if the coefficient $\lambda(t)$ is not consistent with the boundary data, the solution of the obtained via MVI boundary-value problem may not give the desired value of the functional $\mathcal{J}$. Therefore, minimization with respect to the function $\lambda(t)$ should be considered.

It follows from equation (8) that the coefficient $\lambda(t)$ can be computed as

$$
\lambda(t)=\frac{\iint_{\Omega} \frac{\partial T}{\partial t}\left(\frac{\partial^{2} T}{\partial x^{2}}+\frac{\partial^{2} T}{\partial y^{2}}\right) d x d y}{\iint_{\Omega}\left(\frac{\partial^{2} T}{\partial x^{2}}+\frac{\partial^{2} T}{\partial y^{2}}\right)^{2} d x d y} .
$$

This approach does not work for stationary fields.

\section{Existence and Uniqueness of the Solution}

Now we investigate the individual components of the minimization problem for existence and uniqueness of the solution. The uniqueness of each subproblem will not automatically bring uniqueness of the full minimization problem; yet, existence and uniqueness of the subproblems are of crucial importance for constructing algorithms for approximating solutions.

\subsection{Correctness of the Fourth-Order B.V.P.}

In order to prove the correctness of the fourth-order boundary-value problem (10), (4)-(7), we consider the Hilbert space $\mathcal{H}(\mathcal{D})$, where $\mathcal{D}$ is the domain

$\mathcal{D}=\left\{(x, y, t): 0<x<1 ; 0<y<1 ; 0<t<t_{F}\right\}$.

Definition 1 Let $\mathcal{H}$ consists of the functions $\alpha$ satisfying the following boundary conditions

$$
\left.\alpha\right|_{\partial \Omega}=\left.\frac{\partial \alpha}{\partial \nu}\right|_{\partial \Omega}=\alpha(x, y, 0)=\alpha\left(x, y, t_{F}\right)=0,
$$

where $\nu$ is the outward unit normal.

We assume that the functions under consideration are as many time differentiable as necessary.
Definition 2 We introduce the following scalar product in $\mathcal{H}(\mathcal{D})$

$$
\begin{aligned}
{[\alpha, \beta]=} & \int_{0}^{t_{F}} \iint_{\Omega}\left(-\frac{\partial \alpha}{\partial t}+\lambda \frac{\partial^{2} \alpha}{\partial x^{2}}+\lambda \frac{\partial^{2} \alpha}{\partial y^{2}}\right) \\
& \left(-\frac{\partial \beta}{\partial t}+\lambda \frac{\partial^{2} \beta}{\partial x^{2}}+\lambda \frac{\partial^{2} \beta}{\partial y^{2}}\right) d x d y d t
\end{aligned}
$$

where $\lambda(t)>0$ is a function defined for $t>0$.

The equation (13) is a scalar product because for $\lambda(t)>0$, with the homogeneous boundary conditions (12), the equation

$$
-\frac{\partial \alpha}{\partial t}+\lambda \frac{\partial^{2} \alpha}{\partial x^{2}}+\lambda \frac{\partial^{2} \alpha}{\partial y^{2}}=0
$$

has only the trivial solution, i.e. $[\alpha, \alpha]=0$ is true only when $\alpha(x, y) \equiv 0$ in $\mathcal{D}$. The space $\mathcal{H}(\mathcal{D})$, with the scalar product (13), is a Hilbert space.

Let us introduce sufficiently differentiable functions $\chi(x, y, t)$, defined in $\mathcal{D}$, and satisfying the respective boundary conditions (4)-(7). Let us now define the functional

$$
\begin{array}{r}
F(\Phi) \stackrel{\text { def }}{=}-[\chi, \Phi] \\
=-\int_{0}^{t_{F}} \iint_{\Omega}\left(-\frac{\partial \chi}{\partial t}+\lambda \frac{\partial^{2} \chi}{\partial x^{2}}+\lambda \frac{\partial^{2} \chi}{\partial y^{2}}\right) \\
\quad\left(-\frac{\partial \Phi}{\partial t}+\lambda \frac{\partial^{2} \Phi}{\partial x^{2}}+\lambda \frac{\partial^{2} \Phi}{\partial y^{2}}\right) d x d y d t,
\end{array}
$$

where $\Phi \in \mathcal{H}(\mathcal{D})$. Following the Riesz Representation Theorem, for the continuous linear functional $F$ on the Hilbert space $\mathcal{H}$, there is a unique $v \in \mathcal{H}$ such that

$$
F(\Phi)=[v, \Phi]
$$

for all $\Phi \in \mathcal{H}(\mathcal{D})$.

Definition 3 A generalized (weak) solution of the problem (10), (4)-(7) is defined as the function $T:=$ $v+\chi$.

Therefore, the following expression holds true for the weak solution $T$ for all $\Phi \in \mathcal{H}(\mathcal{D})$

$$
\begin{array}{r}
{[T, \Phi]=[T, \Phi]+[\chi, \Phi]=[T, \Phi]-F(\Phi)} \\
=[T, \Phi]-[T, \Phi]=0 .
\end{array}
$$

Theorem 1 The classical solution of the problem (10), (4)-(7) is also a generalized solution.

We multiply equation (10) by $\Phi$, and integrate over the domain $\mathcal{D}$ to obtain 


$$
\begin{gathered}
0=\int_{0}^{t_{F}} \iint_{\Omega} \Phi\left(\frac{\partial}{\partial t}+\lambda \frac{\partial^{2}}{\partial x^{2}}+\lambda \frac{\partial^{2}}{\partial y^{2}}\right) \\
\left(-\frac{\partial T}{\partial t}+\lambda \frac{\partial^{2} T}{\partial x^{2}}+\lambda \frac{\partial^{2} T}{\partial y^{2}}\right) d x d y d t \\
=\int_{0}^{t_{F}} \iint_{\Omega} \Phi \frac{\partial}{\partial t}\left(-\frac{\partial T}{\partial t}+\lambda \frac{\partial^{2} T}{\partial x^{2}}+\lambda \frac{\partial^{2} T}{\partial y^{2}}\right) d x d y d t \\
+\int_{0}^{t_{F}} \iint_{\Omega} \Phi \lambda \frac{\partial^{2}}{\partial x^{2}}\left(-\frac{\partial T}{\partial t}+\lambda \frac{\partial^{2} T}{\partial x^{2}}+\lambda \frac{\partial^{2} T}{\partial y^{2}}\right) d x d y d t \\
+\int_{0}^{t_{F}} \iint_{\Omega} \Phi \lambda \frac{\partial^{2}}{\partial y^{2}}\left(-\frac{\partial T}{\partial t}+\lambda \frac{\partial^{2} T}{\partial x^{2}}+\lambda \frac{\partial^{2} T}{\partial y^{2}}\right) d x d y d t .
\end{gathered}
$$

Using integration by parts we obtain

$$
\begin{aligned}
& \int_{0}^{t_{F}} \iint_{\Omega} \Phi \frac{\partial}{\partial t}\left(-\frac{\partial T}{\partial t}+\lambda \frac{\partial^{2} T}{\partial x^{2}}+\lambda \frac{\partial^{2} T}{\partial y^{2}}\right) d x d y d t \\
& =\iint_{\Omega}\left[\Phi\left(x, y, t_{F}\right)-\Phi(x, y, 0)\right] \\
& \left(-\frac{\partial T}{\partial t}+\lambda \frac{\partial^{2} T}{\partial x^{2}}+\lambda \frac{\partial^{2} T}{\partial y^{2}}\right) d x d y \\
& -\int_{0}^{t_{F}} \iint_{\Omega} \frac{\partial \Phi}{\partial t}\left(-\frac{\partial T}{\partial t}+\lambda \frac{\partial^{2} T}{\partial x^{2}}+\lambda \frac{\partial^{2} T}{\partial y^{2}}\right) d x d y d t \\
& \int_{0}^{t_{F}} \iint_{\Omega} \Phi \lambda \frac{\partial^{2}}{\partial x^{2}}\left(-\frac{\partial T}{\partial t}+\lambda \frac{\partial^{2} T}{\partial x^{2}}+\lambda \frac{\partial^{2} T}{\partial y^{2}}\right) d x d y d t \\
& =\int_{0}^{t_{F}} \int_{0}^{1} \lambda[\Phi(1, y, t)-\Phi(0, y, t)] \\
& \frac{\partial}{\partial x}\left(-\frac{\partial T}{\partial t}+\lambda \frac{\partial^{2} T}{\partial x^{2}}+\lambda \frac{\partial^{2} T}{\partial y^{2}}\right) d y d t \\
& -\int_{0}^{t_{F}} \int_{0}^{1} \lambda\left[\left.\frac{\partial \Phi}{\partial x}\right|_{(1, y, t)}-\left.\frac{\partial \Phi}{\partial x}\right|_{(0, y, t)}\right] \\
& \left(-\frac{\partial T}{\partial t}+\lambda \frac{\partial^{2} T}{\partial x^{2}}+\lambda \frac{\partial^{2} T}{\partial y^{2}}\right) d y d t \\
& +\int_{0}^{t_{F}} \iint_{\Omega} \lambda \frac{\partial^{2} \Phi}{\partial x^{2}}\left(-\frac{\partial T}{\partial t}+\lambda \frac{\partial^{2} T}{\partial x^{2}}+\lambda \frac{\partial^{2} T}{\partial y^{2}}\right) d x d y d t
\end{aligned}
$$

$$
\begin{gathered}
\int_{0}^{t_{F}} \iint_{\Omega} \Phi \lambda \frac{\partial^{2}}{\partial y^{2}}\left(-\frac{\partial T}{\partial t}+\lambda \frac{\partial^{2} T}{\partial x^{2}}+\lambda \frac{\partial^{2} T}{\partial y^{2}}\right) d x d y d t \\
=\int_{0}^{t_{F}} \int_{0}^{1} \lambda[\Phi(x, 1, t)-\Phi(x, 0, t)] \\
\frac{\partial}{\partial y}\left(-\frac{\partial T}{\partial t}+\lambda \frac{\partial^{2} T}{\partial x^{2}}+\lambda \frac{\partial^{2} T}{\partial y^{2}}\right) d x d t \\
-\int_{0}^{t_{F}} \int_{0}^{1} \lambda\left[\left.\frac{\partial \Phi}{\partial y}\right|_{(0,1, t)}-\left.\frac{\partial \Phi}{\partial y}\right|_{(x, 1, t)}\right] \\
+\int_{0}^{t_{F}} \iint_{\Omega} \lambda \frac{\partial T}{\partial t}+\lambda \frac{\partial^{2} \Phi}{\partial y^{2}}\left(-\frac{\partial T}{\partial t}+\lambda \frac{\partial^{2}}{\partial y^{2}}\right) d x d t
\end{gathered}
$$

Since $\Phi \in \mathcal{H}(\mathcal{D})$, all boundary integrals in $(18)$, (19), and (20) vanish. After some manipulations we obtain

$$
\begin{aligned}
& \int_{0}^{t_{F}} \iint_{\Omega}\left(-\frac{\partial \Phi}{\partial t}+\lambda \frac{\partial^{2} \Phi}{\partial x^{2}}+\lambda \frac{\partial^{2} \Phi}{\partial y^{2}}\right) \\
& \left(-\frac{\partial T}{\partial t}+\lambda \frac{\partial^{2} T}{\partial x^{2}}+\lambda \frac{\partial^{2} T}{\partial y^{2}}\right) d x d y d t=[T, \Phi] .
\end{aligned}
$$

Theorem 2 The weak solution of the problem (10), (4)-(7) is unique.

In order to prove the uniqueness, we take the difference $\hat{T}=T_{1}-T_{2}$ between two supposed solutions $T_{1}$ and $T_{2}$. It is obvious that $\hat{T} \in \mathcal{H}(\mathcal{D})$. On the other hand, equation 16 holds true for $\hat{T}$. Then, by taking $\Phi \equiv \hat{T}$, we obtain $[\hat{T}, \hat{T}]=0$, hence $\hat{T} \equiv 0$. Thus, we have shown that the Euler-Lagrange equation (10) possesses a unique solution under the boundary conditions (4)-(7), if the coefficient $\lambda(t)>0$ is given.

\subsection{Correctness of the Problem for the Termal Conductivity $\lambda$}

Since equation (11) is an explicit expression for the coefficient, it provides a unique solution for $\lambda(t)>0$, when the function $T(x, y, t)$ is thought of as known.

\subsection{Existence of Solution of the Full Minimization Problem}

Up to this point, we have shown that the two EulerLagrange equations (10) and (11) possess unique solutions for $T(x, y, t)$ and $\lambda(t)$, provided that the other function is assumed to be known in each equation. This allows one to construct a procedure for finding a solution to the full nonlinear problem by means 


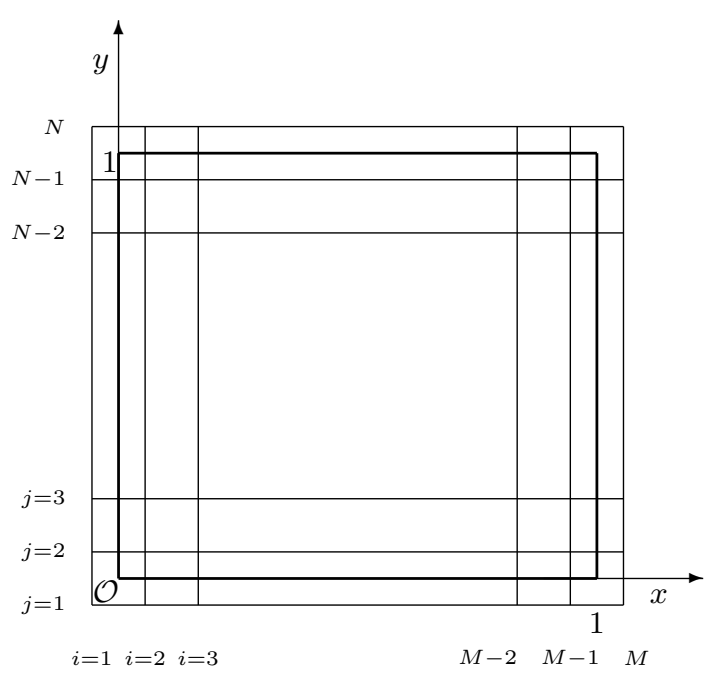

Figure 1: Grid pattern.

of iterations replacing $\lambda(t)$ (when calculating $T$ ), or $T(x, y, t)$ (when calculating $\lambda$ ) with their values calculated from the previous iteration.

If the iterations converge, then they will give one of the possible solutions of the problem. Thus, the existence of the solution to the identification problem can be established a-posteriori. In the light of what has been shown in this section, one can conclude that divergence of the global iteration will necessarily mean that there exists no solution to the identification problem. In other words, for the specified data, there is no function $\lambda(t)$ for which the overposed data stems from the second order parabolic equation (3).

The convergence of the iterations, however, secure only the existence of the solution. It may not be unique, and the iterations can converge to different solutions depending on the initial guess for the functions $T(x, y, t)$ and $\lambda(t)$. Regardless to this limitation, the approach based on MVI is a very useful tool that allows one to find at least one possible coefficient that is consistent with the overposed data. In order to limit the uncertainty of the coefficient estimation, it is possible to incorporate additional restrictions on $\lambda(t)$ based on additional physical information, but they go beyond the framework of the present paper.

\section{Numerical Scheme}

\subsection{Grid and Approximations}

As an example, we consider a problem in the unit square

$$
\mathcal{D}=\{(x, y): 0<x<1 ; 0<y<1\} .
$$

We also introduce an orthogonal mesh with a total number of grid lines equal to $M$ and $N$ in the $x$ - and $y$-directions, respectively. In order to obtain second-order approximation of the derivatives participating in the boundary conditions, we use staggered grid in both directions which overflows the boundaries by half spacing (see Figure 1). The spacings are given by: $h_{x} \equiv 1 /(M-2)$ and $h_{y} \equiv 1 /(N-2)$, and the grid lines are defined by $x_{i}=(i-1.5) h_{x}$ for $i=1, \ldots, M$, and $y_{j}=(j-1.5) h_{y}$ for $j=1, \ldots, N$.

In order to secure second-order approximation in time, we use a non-staggered grid in time for the temperature $T(x, y, t)$. The grid for the coefficient $\lambda(t)$ is staggered (see Figure2).

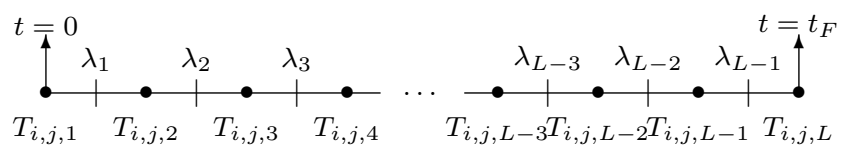

Figure 2: Grid nodes in time.

The total number of grid nodes in the $t$-direction for $T$ is equal to $L$ and the total number of grid nodes in the $t$-direction for $\lambda$ is equal to $L-1$. The spacing in the $t$-direction is $h_{t} \equiv t_{F} /(L-1)$, and the gridnodes for $T$ and $\lambda$ are defined in the following way:

- for $T: t_{l}=(l-1) h_{t}$, for $l=1, \ldots, L$;

- for $\lambda: t_{l+0.5}=(l-0.5) h_{t}$ for $l=1, \ldots, L-1$.

\subsection{Approximation of the Fourth-Order B.V.P.}

The grid described above allows discretization of the derivative in the governing equation and boundary conditions with central differences. The grid-nodes used for approximation of the fourth order partial differential equation (10) are shown at Figure 3. After discretization, we obtain a system of linear equations with the following coefficients: for $T_{i, j, k}$ :

$$
C_{k}^{00}=\frac{2}{h_{t}^{2}}+\frac{6 l_{k}^{2}}{h_{x}^{4}}+\frac{8 l_{k}^{2}}{h_{x}^{2} h_{y}^{2}}-\frac{2 l_{k}^{\prime}}{h_{x}^{2}}+\frac{6 l_{k}^{2}}{h_{y}^{4}}-\frac{2 l_{k}^{\prime}}{h_{y}^{2}} ;
$$

for $T_{i, j-1, k}, T_{i, j+1, k}$ :

$$
C_{k}^{01}=-\frac{4 l_{k}^{2}}{h_{x}^{2} h_{y}^{2}}-\frac{4 l_{k}^{2}}{h_{y}^{4}}+\frac{l_{k}^{\prime}}{h_{y}^{2}}
$$

for $T_{i, j-2, k}, T_{i, j+2, k}$ :

$$
C_{k}^{02}=\frac{l_{k}^{2}}{h_{y}^{4}}
$$

for $T_{i-1, j-1, k}, T_{i-1, j+1, k}, T_{i+1, j-1, k}, T_{i+1, j+1, k}$ :

$$
C_{k}^{11}=\frac{2 l_{k}^{2}}{h_{x}^{2} h_{y}^{2}}
$$




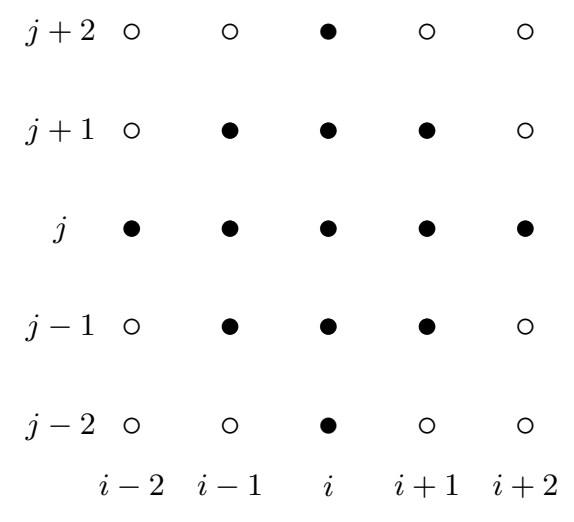

Figure 3: Grid nodes $(\bullet)$ used in approximation of the derivatives with respect to $x$ and $y$.

for $T_{i-1, j, k}, T_{i+1, j, k}$ :

$$
C_{k}^{10}=-\frac{4 l_{k}^{2}}{h_{x}^{4}}-\frac{4 l_{k}^{2}}{h_{x}^{2} h_{y}^{2}}+\frac{l_{k}^{\prime}}{h_{x}^{2}} ;
$$

for $T_{i-2, j, k}, T_{i+2, j, k}$ :

$$
C_{k}^{20}=\frac{l_{k}^{2}}{h_{x}^{4}}
$$

for $T_{i, j, k-1}, T_{i, j, k+1}$ :

$$
-\frac{1}{h_{t}^{2}}
$$

\subsection{Approximation of the Thermal Conductivity $\lambda$}

Let us introduce the following notations

$$
\begin{aligned}
\Lambda_{x x}^{i, j, k}=\frac{T_{i-1, j, k}-}{2 T_{i, j, k}+T_{i+1, j, k}} & h_{x}^{2} \\
= & \left.\frac{\partial^{2} T}{\partial x^{2}}\right|_{\left(x_{i}, y_{j}, t_{k}\right)}+O\left(h_{x}^{2}\right), \\
\Lambda_{y y}^{i, j, k}=\frac{T_{i, j-1, k}-}{2 T_{i, j, k}+T_{i, j+1, k}} & h_{y}^{2} \\
= & \left.\frac{\partial^{2} T}{\partial y^{2}}\right|_{\left(x_{i}, y_{j}, t_{k}\right)}+O\left(h_{y}^{2}\right),
\end{aligned}
$$

for $i=2,3, \ldots, M-1, j=2,3, \ldots, N-1$, and $k=1,2, \ldots, L$. Also,

$$
\begin{aligned}
& \Lambda_{t}^{i, j, k}=\frac{T_{i, j, k+1}-T_{i, j, k}}{h_{t}} \\
&=\left.\frac{\partial T}{\partial t}\right|_{\left(x_{i}, y_{j}, t_{k+1 / 2}\right)}+O\left(h_{t}^{2}\right),
\end{aligned}
$$

for $i=2,3, \ldots, M-1, j=2,3, \ldots, N-1$, and $k=1,2, \ldots, L-1$.

We use the extended Midpoint rule to approximate the integrals in (11) of the second-order with respect to all variables:

$$
\begin{aligned}
& S_{1, k}= \frac{h_{x} h_{y}}{2} \sum_{i=2}^{M-1} \sum_{j=2}^{N-1} \\
& \Lambda_{t}^{i, j, k}\left(\Lambda_{x x}^{i, j, k}+\Lambda_{x x}^{i, j, k+1}+\Lambda_{y y}^{i, j, k}+\Lambda_{y y}^{i, j, k+1}\right) \\
&=\iint_{\Omega} \frac{\partial T}{\partial t}\left(\frac{\partial^{2} T}{\partial x^{2}}+\frac{\partial^{2} T}{\partial y^{2}}\right) d x d y \\
& \quad+O\left(h_{x}^{2}+h_{y}^{2}+h_{t}^{2}\right)
\end{aligned}
$$

$$
\begin{aligned}
& S_{2, k}=\frac{h_{x} h_{y}}{4} \sum_{i=2}^{M-1} \sum_{j=2}^{N-1} \\
&\left(\Lambda_{x x}^{i, j, k}+\Lambda_{x x}^{i, j, k+1}+\Lambda_{y y}^{i, j, k}+\Lambda_{y,}^{i, j, k+1}\right)^{2} \\
&=\iint_{\Omega}\left(\frac{\partial^{2} T}{\partial x^{2}}+\frac{\partial^{2} T}{\partial y^{2}}\right)^{2} \\
& d x d y+O\left(h_{x}^{2}+h_{y}^{2}+h_{t}^{2}\right),
\end{aligned}
$$

for $k=1,2, \ldots, L-1$. The equation (11) is approximated as

$$
\lambda_{k}=\frac{S_{1, k}}{S_{2, k}} .
$$

\subsection{Algorithm}

The iterations are performed as follows:

(I) With a given initial values for $\lambda_{k}^{\text {old }}>0$, the fourthorder boundary value problem equations(10), (5), (7) is solved for the function $T_{i, j, k}$.

(II) With the newly computed values of $T_{i, j, k}$, the function $\lambda_{k}^{\text {new }}$ is evaluated.

If the norm of the difference between the new and the old field for $\lambda$ is less than $\varepsilon$, i.e. $\max _{k} \mid \lambda_{k}^{\text {new }}-$ $\lambda_{k, l}^{\text {old }} \mid<\varepsilon$, then the calculations are terminated, otherwise step (I) is repeated.

\section{Validation of the Algorithm}

We check the accuracy of the difference scheme for the fourth-order problem using tests involving different grid spacing $h_{x}, h_{y}$, and $h_{t}$. We do that to verify that the solution of the linear imbedding problem for the unknown function does coincide with the solution of the "direct" problem for a given coefficient. We used different expressions for the coefficient, including the constant. The findings are essentially the same. We present here the results with a coefficient 


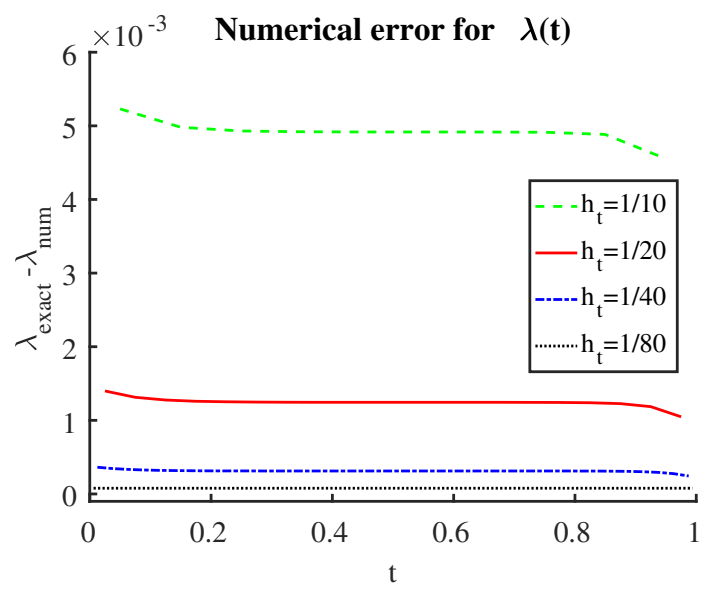

Figure 4: The difference between numerical and exact values of $\lambda(t)$ for $h_{x}=h_{y}=h_{t}=$ $0.1,0.05,0.025,0.0125$ for $\lambda=0.5$.

Table 1: The $l^{2}$ norm of the difference $\lambda-\lambda_{\text {exact }}$, and the rates of convergence for four different values of the grid spacings $h_{x}=h_{y}=h_{t}$ for $\lambda=0.5$.

\begin{tabular}{|c|c|c|}
\hline$h_{t}$ & $\| \lambda-\lambda_{\text {exact }} \mid l_{l^{2}}$ & rate $_{\lambda}$ \\
\hline 0.100000 & 0.004918 & - \\
\hline 0.050000 & 0.001246 & 1.980654 \\
\hline 0.025000 & 0.000312 & 1.995780 \\
\hline 0.012500 & 0.000078 & 2.002137 \\
\hline \hline
\end{tabular}

that is a constant, continuous nonlinear function of time, and discontinuous function of time.

We also check the rates of convergence calculated as

$$
\operatorname{rate}_{\lambda}=\log _{2} \frac{\left\|\lambda_{2 h}-\lambda_{\text {exact }}\right\|}{\left\|\lambda_{h}-\lambda_{\text {exact }}\right\|} .
$$

\subsection{Constant Coefficient}

The temperature and the coefficient in this case are as follows

$$
T(x, y, t)=e^{-4 t} \sin (2 x+2 y) \text {, and } \lambda(t)=0.5 .
$$

Under proper boundary conditions the numerical solution has to converge to the exact solution (29). Figure 4 shows the point-wise numerical errors for the function $\lambda(t)$ calculated with for different spacings $h_{x}=h_{y}=h_{t}=0.1 ; 0.05 ; 0.025 ; 0.0125$.

The $l_{2}$ norm of the difference between the numerical solution $\lambda$ and the analytical solution (29) with four different grid spacings $h_{x}=h_{y}=h_{t}=$ $0.1 ; 0.05 ; 0.025 ; 0.0125$ is given in Table 1 along with the rates of convergence. The presented numerical tests confirm the second order rate of convergence.

\subsection{Continuous Nonlinear Coefficient}

In order to prove numerically the second-order of approximation, consider the following exact solution of

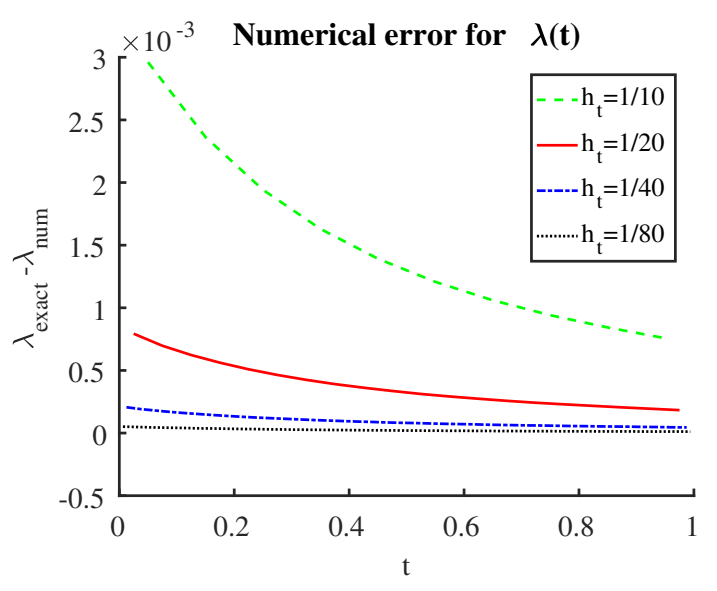

Figure 5: The difference between numerical and exact values of $\lambda(t)$ for $h_{x}=h_{y}=h_{t}=$ $0.1,0.05,0.025,0.0125$ for $\lambda(t)=\frac{1}{1+t}$.

Table 2: The $l^{2}$ norm of the difference $\lambda-\lambda_{\text {exact }}$, and the rates of convergence for four different values of the grid spacings $h_{x}=h_{y}=h_{t}$ for $\lambda(t)=\frac{1}{1+t}$.

\begin{tabular}{|c|c|c|}
\hline$h_{t}$ & $\left\|\lambda-\lambda_{\text {exact }}\right\| l_{l^{2}}$ & rate $_{\lambda}$ \\
\hline 0.100000 & 0.001657 & - \\
0.050000 & 0.000416 & 1.993292 \\
0.025000 & 0.000104 & 1.998393 \\
0.012500 & 0.000026 & 2.002052 \\
\hline
\end{tabular}

the equation (2):

$$
T(x, y, t)=(1+t)^{2} e^{-(x+y)}, \quad \lambda(t)=\frac{1}{1+t} .
$$

Under proper boundary conditions the numerical solution has to converge to the exact one in (30).

Figure 5 shows the point-wise numerical errors for the function $\lambda(t)$ calculated with different spacings $h_{x}=h_{y}=h_{t}=0.1 ; 0.05 ; 0.025 ; 0.0125$.

The $l_{2}$ norm of the difference between the numerical solution $\lambda$ and the analytical solutions using the four different grid spacings is given in Table 2 along with the rates of convergence. The presented numerical tests demonstrate the second order rate of convergence. The most important point here is that in this case we found that the identification of the coefficient is unique in the sense that we started the iterative process from rather different initial conditions and in all cases we ended up with the same numerical solution, whose error is depicted in Figure 5.

\subsection{Discontinuous Coefficient}

We now choose a rather hard test case with a coefficient to be identified being a discontinuous function, 


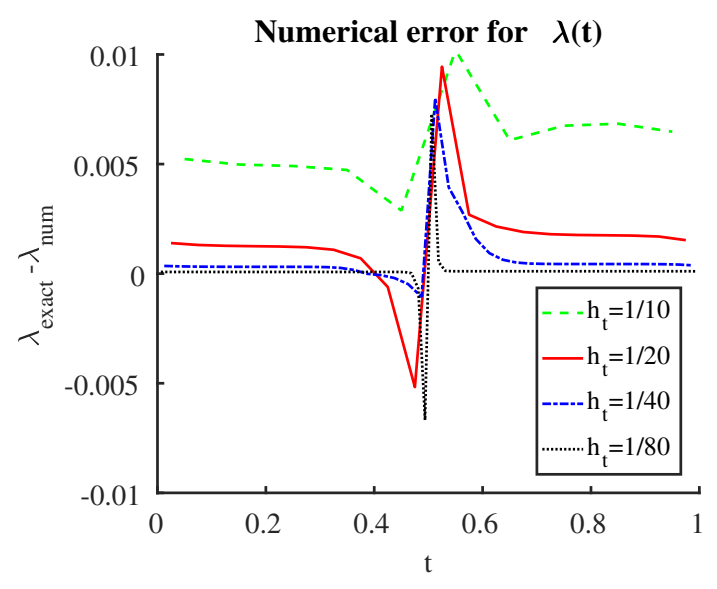

Figure 6: The difference between numerical and exact values of $\lambda(t)$ for $h_{x}=h_{y}=h_{t}=$ $0.1,0.05,0.025,0.0125$ for $\lambda(t)$ given with equation (31).

namely

$$
\lambda(t)= \begin{cases}0.5 & \text { if } t<0.5 \\ 0.55 & \text { if } t>0.5\end{cases}
$$

The temperature distribution $T$ is

$T(x, y, t)= \begin{cases}e^{(-4 t+2)} \sin (2 x+2 y) & \text { if } t \leq 0.5 \\ e^{(-4.4 t+2.2)} \sin (2 x+2 y) & \text { if } t>0.5 .\end{cases}$

Under the corresponding boundary conditions the numerical solution has to converge to the exact one. However, because of singularity, we cannot expect the second order of convergence.

Fig. 6 shows the point-wise numerical errors for the function $\lambda(t)$ calculated with for different spacings $h_{x}=h_{y}=h_{t}=0.1 ; 0.05 ; 0.025 ; 0.0125$.

The $l_{1}$ norm of the difference between the numerical solution $\lambda$ and the analytical solution with four different grid spacings $h_{x}=h_{y}=h_{t}=$ $0.1 ; 0.05 ; 0.025 ; 0.0125$ is given in Table 3 along with the rates of convergence. The presented numerical tests demonstrate rate of convergence about 1.5. The reason for this behavior is the singularity at the plane $t=0.5$. The numerical error for the temperature, $\frac{T_{\text {exact }}-T_{\text {numeric }}}{T_{\text {exact }}}$, at the plane $x=0.5$ for $h_{x}=h_{y}=h_{t}=$ 0.0125 is shown at Figure 7 .

\section{Conclusions}

This paper proposes an approach for solving the inverse coefficient problem for the (2+1)D heat conduction equation based on the measurements of temperature and heat flux at the body's boundary. The original inverse problem is replaced by a minimization problem. The Euler-Lagrange equations for minimization

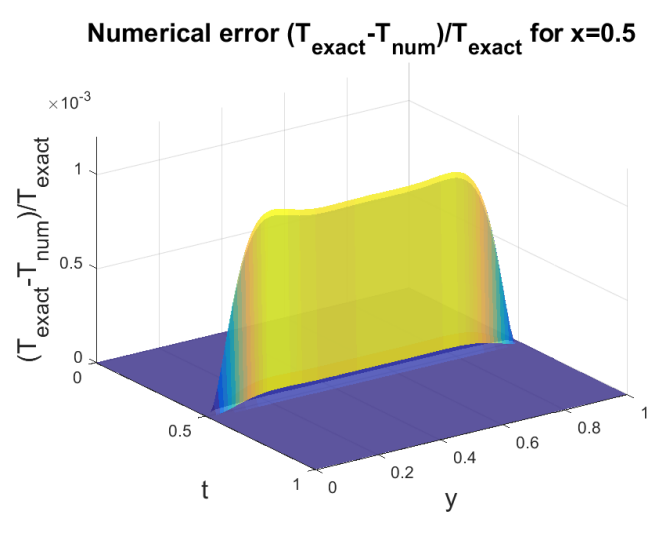

Figure 7: The difference between numerical and exact values of $T$ for $h_{x}=h_{y}=h_{t}=0.0125$ at the plane $x=0.5$.

Table 3: The $l^{1}$ norm of the difference $\lambda-\lambda_{\text {exact }}$, and the rates of convergence for four different values of the grid spacings $h_{x}=h_{y}=h_{t}$ for $\lambda(t)$ given with equation (31).

\begin{tabular}{|c|c|c|}
\hline$h_{t}$ & $\left\|\lambda-\lambda_{\text {exact }}\right\|_{l^{1}}$ & $\operatorname{rate}_{\lambda}$ \\
\hline 0.100000 & 0.005909 & - \\
\hline 0.050000 & 0.002086 & 1.502476 \\
\hline 0.025000 & 0.000761 & 1.454726 \\
\hline 0.012500 & 0.000278 & 1.450635 \\
\hline \hline
\end{tabular}

comprise a fourth-order elliptic equation for the temperature, the function $T=T(x, y, t)$, and an explicit equation for the unknown coefficient $\lambda=\lambda(t)$. For this system, the boundary data are no longer overposed. Thus, the original inverse problem is embedded into a higher-order but well-posed problem. The numerical simulations show that the proposed method gives an accurate simultaneous identification of the temperature and the thermal conductivity.

\section{References:}

[1] Brociek R., Słota D., Wituła R., Reconstruction of the Thermal Conductivity Coefficient in the Time Fractional Diffusion Equation. In: Latawiec K., Łukaniszyn M., Stanisławski R. (eds) Advances in Modelling and Control of Noninteger-Order Systems. Lecture Notes in Electrical Engineering, Springer, Cham 320, (2015), pp. 239-247

[2] Brociek R., Słota D., Reconstruction of the thermal conductivity coefficient in the space fractional heat conduction equation, Thermal Science 21 (1 Part A), (2017), pp. 81-88

[3] Christov C. I., Marinov T. T., Identification of Heat-conduction Coefficient via Method of 
Variational Imbedding, Mathematical and Computer Modelling, 27 (3) (1998), pp. 109-116

[4] Engl H.W., Zou J., A new approach to convergence rate analysis of Tikhonov regularization for parameter identification in heat conduction, Inverse Problems, 16 (2000), pp. 1907-1923

[5] Hadała B., Malinowski Z., Szajding A., Solution strategy for the inverse determination of the specially varying heat transfer coefficient, International Journal of Heat and Mass Transfer, 104 (2017), pp. 993-1007

[6] Hadamard J., Le Probleme de Cauchy et les Equations aux Derivatives Partielles Lineares Hyperboliques, Hermann, Paris (1932)

[7] Hristov J., An approximate solution to the transient space-fractional diffusion equation: Integral-balance approach, optimization problems and analyzes, Thermal Science 21(1 Part A) (2016), pp. 309-321

[8] Huntul M.J., Hussein M.S., Lesnic D., Ivanchov M.I., Kinash N., Reconstruction of an orthotropic thermal conductivity from non-local heat flux measurements, International Journal of Mathematical Modelling and Numerical Optimisation, 10(1) pp. 102-122 (2020)

[9] Kakac S., Yener Y., Naveira-Cotta C. P., Heat Conduction. 5th Edition. CRC Press, Boca Raton (2018)

[10] Kolehmainen V., Kaipio J.P., Orlande H.R.B., Reconstruction of thermal conductivity and heat capacity using a tomographic approach, International Journal of Heat and Mass Transfer 50(2526) (2007), pp. 5150-5160

[11] Karageorghis A., Lesnic D., Steady-state nonlinear heat conduction in composite materials using the method of fundamental solutions, Computer Methods in Applied Mechanics and Engineering, 197(33-40), 2008, pp. 3122-3137

[12] Kügler P., Identification of a Temperature Dependent Heat Conductivity from Single Boundary Measurements, SIAM J. Numer. Anal, 41 (4) (2004), pp. 1543-1563

[13] Lesnic D., Elliott L., Ingham D. B., Identification of the Thermal Conductivity and Heat Capacity in Unsteady Nonlinear Heat Conduction
Problems Using the Boundary Element Method, Journal of Computational Physics, 126 (1996), pp. $410-420$

[14] Malinowski Z., Cebo-Rudnicka A., Hadała B., Szajding A., Inverse method implementation to heat transfer coefficient determination over the plate cooled by water spray, Inverse Problems in Science and Engineering, 23(3) (2014), pp $518-556$

[15] Marinov T. T., Christov C. I., Marinova R. S., Novel numerical approach to solitarywave solutions identification of Boussinesq and Korteweg-de Vries equations, Int. J. of Bifurcation and Chaos 15 (2) (2005), 557-565

[16] Marinov T. T., Marinova R. S., Christov C. I., Coefficient Identification in Elliptic Partial Differential Equation, In: Lirkov I., Margenov S., Waniewski J. (eds) Large-Scale Scientific Computing. LSSC 2005. Lecture Notes in Computer Science, Springer, Berlin, Heidelberg 3743 (2006), pp. 372-379

[17] Marinov T.T., Marinova R.S., Identification of Heat Conductivity in $(2+1) \mathrm{D}$ Equation as a Function of Time, Large Scale Scientific Computing, Lecture Notes in Computer Science, Springer (2020), pp. 356-364

[18] Nedin R., Nesterov S., Vatulyan A., Identification of thermal conductivity coefficient and volumetric heat capacity of functionally graded materials, International Journal of Heat and Mass Transfer, 102 (2016), pp. 213-218

[19] Szénási S., Felde I., Using multiple graphics accelerators to solve the two-dimensional inverse heat conduction problem, Computer Methods in Applied Mechanics and Engineering, 336 (2018), pp. 286-303

[20] Tikhonov A.N. and Arsenin V.Y., Methods for Solving Incorrect Problems, Nauka, Moscow (1974)

[21] Xiong X.T., Liu X.H., Yan Y.M., Guo H.B., A numerical method for identifying heat transfer coefficient, Applied Mathematical Modelling, Vol. 34 (7) (2010), pp. 1930-1938

\section{Creative Commons Attribution License 4.0 (Attribution 4.0 International, CC BY 4.0)}

This article is published under the terms of the Creative Commons Attribution License 4.0

https://creativecommons.org/licenses/by/4.0/deed.en_US 